\title{
Nonlinear optical probing of nanocrystalline orientation in epitaxial ferroelectric thin films
}

\author{
G. Woltersdorf, ${ }^{\text {a) }}$ G. Seifert, ${ }^{\text {b) }}$ and H. Graener \\ Fachbereich Physik, Martin-Luther-Universität Halle-Wittenberg, D-06099 Halle, Germany
}

A Pignolet and D. Hesse

Max-Planck-Institut für Mikrostrukturphysik, Weinberg 2, D-06120 Halle, Germany

(Received 29 May 2001; accepted for publication 26 September 2001)

\begin{abstract}
Epitaxially grown ferroelectric $\mathrm{BaBi}_{4} \mathrm{Ti}_{4} \mathrm{O}_{15}$ thin films were studied by optical second-harmonic (SH) generation using a femtosecond titanium:sapphire laser at $800 \mathrm{~nm}$ wavelength. By varying both the incidence and the azimuthal angle and registering the second-harmonic intensity, a significant correlation was found between the azimuthal dependence of the measured SH signal and the nanoscopic texture of the samples, which was determined by electron microscopy and $\mathrm{x}$-ray diffraction. In particular, two different types of grains with different crystallographic orientations generate different SH intensity maxima with fourfold azimuthal symmetry, distinguishable from an isotropic background due to a $c$-axis oriented main layer. This correlation suggests the use of the $\mathrm{SH}$ technique for a purely optical characterization of the film orientation and thickness. (C) 2002 American Institute of Physics. [DOI: 10.1063/1.1420764]
\end{abstract}

\section{INTRODUCTION}

Ferroelectric thin films are currently being extensively studied both because of their various existing and potential applications and because of many interesting fundamental aspects inherent in these materials. ${ }^{1-3}$ Thin films of $\mathrm{Pb}(\mathrm{Zr}, \mathrm{Ti}) \mathrm{O}_{3}(\mathrm{PZT})$ and $\mathrm{SrBi}_{2} \mathrm{Ta}_{2} \mathrm{O}_{9}$ (SBT) are already in use in commercial products, and SBT thin films, since they do not suffer from fatigue, ${ }^{4,5}$ are particularly promising as the functional elements of ferroelectric random access memories (FeRAMs). ${ }^{1,6,7}$ This advantage of SBT films is a result of the unique crystal structure of the bismuth-layered perovskites (also called Aurivillius phases). The unit cell of the lattice of this type of compound consists of perovskite blocks sandwiched between $\mathrm{Bi}_{2} \mathrm{O}_{2}$ layers, ${ }^{8-10}$ and can be described by the general formula $\left(\mathrm{Bi}_{2} \mathrm{O}_{2}\right)^{2+}\left(\mathrm{A}_{n-1} \mathrm{~B}_{n} \mathrm{O}_{3 n+1}\right)^{2-}$, where $n$, called the Aurivillius parameter, is the number of oxygen octahedra between $\mathrm{Bi}_{2} \mathrm{O}_{2}$ layers. The direction of spontaneous polarization in bismuth-layered compounds has been reported to depend on the number, $n-1$, of perovskite blocks between the $\mathrm{Bi}_{2} \mathrm{O}_{2}$ layers, and on whether it is even or odd. ${ }^{9,11-16}$ Bismuth-layered perovskites with an even Aurivillius parameter have no component of spontaneous polarization along the crystallographic $c$ axis, whereas those which have an odd Aurivillius parameter do. However, irrespective of the Aurivillius parameter spontaneous polarization has a large component of polarization along the crystallographic $a$ axis.

In recent years optical second-harmonic generation was shown to be a powerful method by which to investigate the crystallographic orientation and ferroelectric domain structure of materials such as $\mathrm{PbTiO}_{3},{ }^{17} \mathrm{BaTiO}_{3},{ }^{18-20}$ or

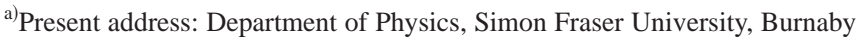
BC, Canada.

${ }^{b)}$ Corresponding author; electronic mail: g.seifert@physik.uni-halle.de
}

$\mathrm{Bi}_{4} \mathrm{Ti}_{3} \mathrm{O}_{12} \cdot{ }^{21}$ In this article the second order nonlinear optical properties of epitaxial ferroelectric thin films of $\mathrm{BaBi}_{4} \mathrm{Ti}_{4} \mathrm{O}_{15}$ (BBiT) are reported, a material with $A=(\mathrm{Ba}, \mathrm{Bi}), B=\mathrm{Ti}, n$ $=4$ and strong crystallographic anisotropy. ${ }^{22,23}$ Therefore, the ferroelectric properties, the remnant polarization as well as the dielectric properties also depend on the crystallographic direction, i.e., for epitaxial thin films, on their orientation with respect to the substrate normal. The crystal structure of BBiT is pseudotetragonal (actually the true symmetry is orthorhombic), with lattice parameters $a=0.5459 \mathrm{~nm}$ and $c=4.185 \mathrm{~nm}^{8}{ }^{8}$ Measurements of the dielectric properties of single crystal BBiT and their anisotropy have recently been reported, ${ }^{24}$ as has the anisotropy in the ferroelectric properties of BBiT thin films. ${ }^{22,23}$

Since the growth mode and film orientation critically depend on many deposition parameters which are not all accessible and/or adjustable, it is highly desirable to have an in situ technique which provides information on the orientation and film thickness during deposition. In this article we report second-harmonic generation (SHG) in BBiT ferroelectric thin films that indicates that SHG could be an ideal nondestructive optical in situ technique by which to control the film orientation and thickness during growth.

\section{FILM DEPOSITION AND PROPERTIES}

The BBiT films were deposited on epitaxial $\mathrm{LaNiO}_{3}$ (LNO) conductive layers that serve both as epitaxial templates and as bottom electrodes. The BBiT and LNO films were grown by pulsed laser deposition (PLD) on singlecrystal $\mathrm{Si}$ (100) coated with epitaxial YSZ and $\mathrm{CeO}_{2}$ buffer layers using a $\mathrm{KrF}$ excimer laser $(\lambda=248 \mathrm{~nm})$ at a laser repetition rate of $5 \mathrm{~Hz}$ with a laser pulse energy density of 2 $\mathrm{J} / \mathrm{cm}^{2}$. The epitaxial quality of the films and their orientation were studied by $\mathrm{x}$-ray diffraction and cross-sectional transmission electron microscopy (XTEM). The film morphology 

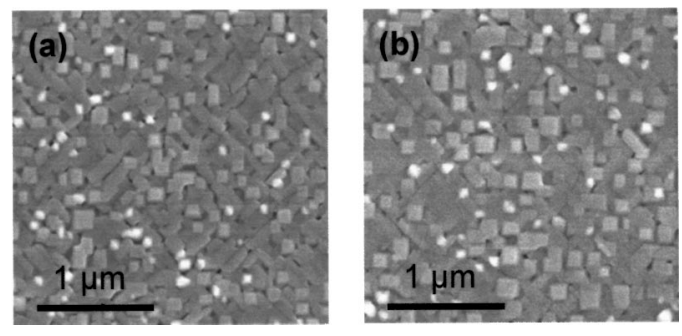

FIG. 1. SEM pictures of $2.7 \times 2.7 \mu \mathrm{m}^{2}$ sq areas of a $\mathrm{BaBi}_{4} \mathrm{Ti}_{4} \mathrm{O}_{15}$ film where (a) the density of rectangular-shaped crystallites is higher than that of square-shaped crystallites and (b) the density of square-shaped crystallites is higher than that of rectangular-shaped crystallites.

was probed by scanning electron microscopy (SEM) and atomic force microscopy (AFM), and ferroelectric measurements were carried out on planar capacitor structures using an RT66A ferroelectric tester. Details of the LNO and BBiT thin film deposition and their properties are reported elsewhere. $^{22,23}$

BBiT thin films mainly grow $c$ oriented on (100) oriented epitaxial LNO electrodes, with a small fraction of the films growing (100) and (110) oriented, as demonstrated by $\mathrm{x}$-ray diffraction studies. Morphology investigations by SEM and AFM showed that more or less rectangular- and squareshaped crystallites protrude from a rather flat background. Detailed TEM studies on both plan-view and cross-sectional samples revealed the following details about their orientation: the flat background is $c$ axis oriented, i.e., its [001] axis is normal to the film plane. The rectangular- and squareshaped crystallites are embedded into this $c$-axis oriented background, leaving a thin uniformly $c$-axis oriented layer directly on the LNO layer. The rectangular-shaped crystallites have the [110] axis normal to the film plane, hereafter called (110) orientation, whereas the [100] axis is normal to the film plane in the square-shaped crystallites, i.e., the latter are (100) oriented. The (110)-oriented rectangular grains have two definite azimuthal orientations. As a result their long edges are parallel to one of two mutually perpendicular directions in the substrate plane. The edges of the squareshaped crystallites form an angle of $45^{\circ}$ with respect to the edges of the rectangle-shaped crystallites.

The areal density of the rectangular- and squared-shaped crystallites is not at all uniform over entire area of the sample (Fig. 1). Ferroelectric hysteresis measurements were carried out on the BBiT films, separately accessing different regions that showed different areal densities of embedded crystallites. In the regions with embedded (110)- and (100)-oriented crystallites the BBiT films exhibit saturated ferroelectric loops with remnant polarization of $2 \mu \mathrm{C} / \mathrm{cm}^{2}$ and a coercive field of $60 \mathrm{kV} / \mathrm{cm}$. In contrast, no hysteresis loops were observed in smooth regions consisting of only the $c$-axis oriented background. This observation reveals the anisotropy of the ferroelectric properties of BBiT and shows that macroscopic polarization depends on the concentration of the non$c$-oriented crystallites in the film. The polarization measured clearly depends on the crystalline orientation of the epitaxial film, and the results demonstrate that BBiT possesses no spontaneous polarization along its crystallographic $c$ axis, in very good agreement with recently published work. ${ }^{13,15,16}$

\section{SECOND-HARMONIC GENERATION}

\section{A. Experimental setup}

The experimental setup for the nonlinear optical experiments is shown schematically in Fig. 2. The output of a mode-locked titanium:sapphire laser (Spectra Physics, Tsunami, pulse duration $80 \mathrm{fs}$, pulse energy $6 \mathrm{~nJ}$, repetition rate $82 \mathrm{MHz}$, wavelength $800 \mathrm{~nm}$ ) passes a polarizer (P1) to ensure linear polarization (for all experiments shown the polarization was in the plane of incidence) and a filter combination (F1) to block any second-harmonic light which may be generated in the previous optical path. This "clean" laser fundamental light is focused onto the sample to a beam waist of approximately $100 \mu \mathrm{m}$; the reflected beam containing the second-harmonic wave generated is then collimated again. While the fundamental frequency is rejected by filter F2, the second harmonic is split into its polarization components parallel and perpendicular to the plane of incidence ( $p$ and $s$ components), which are then registered simultaneously using two channel plate photomultipliers. Modulation of the fundamental wave at $3 \mathrm{kHz}$ using a chopper $(\mathrm{CH})$ allows lock-in detection of the SH signal.

The sample and the detection setup are mounted on three step motor-driven rotation tables: the first allows sample rotation around the surface normal (azimuthal angle $\phi$ ). The second table adjusts the incidence angle, $\theta$, while the third table rotates the complete detection unit by $2 \theta$ with respect to the $k$ vector of the incoming laser light to ensure the reflection condition for the observation. In addition the sample holder can be tilted and shifted for optimal adjustment, i.e., to keep the conditions so that laser focus and rotation axis coincide and the reflected beam does not change direction during rotation. With the described setup the incidence angle $\theta$ can be varied from $10^{\circ}$ to $70^{\circ}$, where the only limitations are simply the dimensions of the optical elements and the sample holder, respectively. Because the azimuthal angle $\phi$ can be varied $360^{\circ}$ the setup described allows one to measure quite comprehensively the angle and polarization dependence of the SHG.

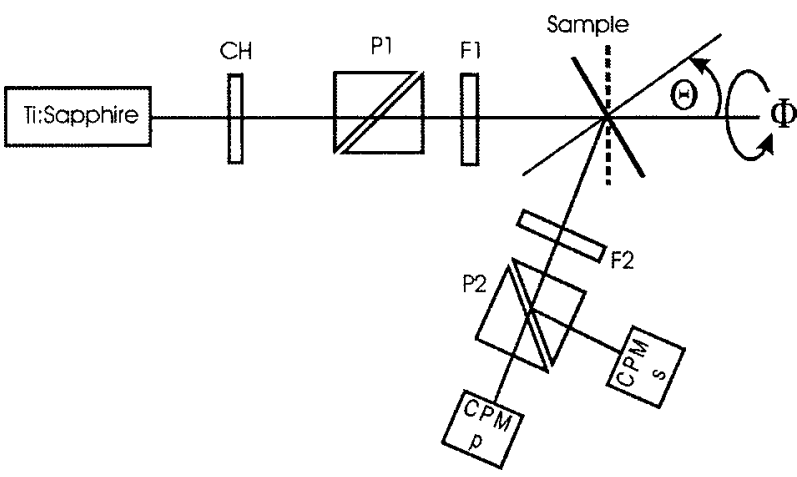

FIG. 2. Schematic of the setup for the SHG experiments: CH: chopper; P1, $\mathrm{P} 2$ : polarizers; F1, F2: filters; CPM $p / s$ : channel plate photomultiplier for registration of the $p / s$ polarization component. 


\section{B. Description of the signal measured}

Illuminating a sample of thickness $l_{s}$ with an intensity $I(\omega)$ generates in general a second-harmonic intensity $I_{s}^{(2 \omega)}$, at least for materials without inversion symmetry. This intensity can be related to the sample parameters by

$$
I_{s}^{(2 \omega)} \propto l_{s}^{2} I^{2}(\omega) \frac{d_{\mathrm{eff}}}{n_{s}(2 \omega) n_{s}^{2}(\omega)} \frac{\sin ^{2}\left(\pi l_{s} / 2 l_{c}\right)}{\left(\pi l_{s} / 2 l_{c}\right)^{2}} .
$$

Here $n_{s}(\omega)$ and $n_{s}(2 \omega)$ are the sample refractive indices at the fundamental and the second-harmonic frequencies, respectively, $d_{\text {eff }}$ is the effective nonlinearity of the sample and $l_{c}$ the coherence length for second-harmonic generation defined as

$$
l_{c}=\frac{\pi c}{2 \omega} \frac{1}{\left[n_{s}(2 \omega)-n_{s}(\omega)\right]} .
$$

As for the investigated ferroelectric films $l_{c}$ is of the order of several micrometers but the film thickness $l_{s}$ $<300 \mathrm{~nm}$, the phase matching factor $\sin ^{2} x / x^{2}$ in Eq. (1) can be set to unity, i.e., phase matching need not be considered in this work. On the other hand, this means that no amplification by phase effects can be utilized to determine absolute values of the effective second order optical nonlinearity $d_{\text {eff }}$. Also, the standard method for quantitative determination of $d_{\text {eff }}$ with the help of a quartz crystal reference ${ }^{25,26}$ cannot simply be used in our case of reflection geometry. So we refrain from trying to determine absolute values of $d_{\text {eff }}$, and will only discuss the angular dependence of the SH intensity measured in the following. To this end it is necessary to calculate the net polarization at the second-harmonic frequency $P^{(2 \omega)}$. In the coordinate system of the crystal (indices $i, j$, and $k$ ) polarization is a function of the incident electric field of the fundamental wave $E^{\omega}$ and of the second order nonlinearity $d$ :

$$
P_{i}^{(2 \omega)}=2 d_{i j k} E_{j}^{\omega} E_{k}^{\omega} .
$$

The transformation of the nonlinear susceptibility in the laboratory coordinate system (indices $p, q$, and $r$ ) is done by $^{27}$

$$
d_{p q r}=\sigma_{p i} \sigma_{q j} \sigma_{r k} d_{i j k},
$$

where $\sigma$ is the rotation matrix containing the sample orientation angles $\phi$ and $\theta$ (see Fig. 2).

The tensor $d$ is usually simplified with the help of symmetry considerations and given in contracted form as a $3 \times 6$ matrix, where additionally several tensor elements may reduce to zero, and the nonzero ones are often not independent of each other. ${ }^{28,29}$ In the particular case of the $m m 2$ symmetry of the BBiT crystal structure investigated only three independent nonzero elements $\left(d_{15}, d_{24}\right.$ and $\left.d_{33}\right)$ remain. Using Eqs. (3) and (4) the second order nonlinear polarization can be evaluated by its projections parallel and perpendicular to the $p$ polarization of the incident field yielding

$$
\begin{aligned}
& P_{\|}^{2 \omega}=\left(P_{x}^{2 \omega} \cos \phi+P_{y}^{2 \omega} \sin \phi\right) \cos \theta+P_{z}^{2 \omega} \sin \theta, \\
& P_{\perp}^{2 \omega}=P_{x}^{2 \omega} \sin \phi-P_{y}^{2 \omega} \cos \phi,
\end{aligned}
$$

with the following abbreviations:
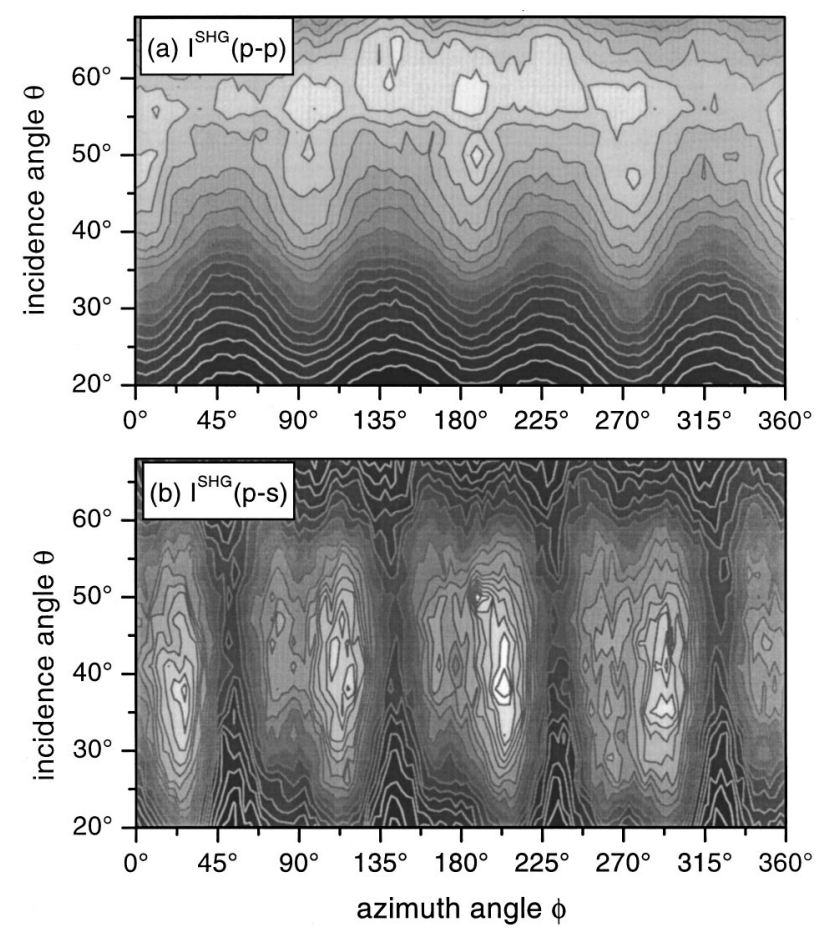

FIG. 3. Gray scale plot of the $\mathrm{SH}$ intensity (at $\lambda=400 \mathrm{~nm}$ ) from a BBiT sample as a function of azimuth angle $\phi$ and incidence angle $\theta$ that refers to (a) $p-p$ geometry (incident laser and $\mathrm{SH}$ observation in the plane of incidence) and (b) $p-s$ geometry.

$$
\begin{aligned}
P_{x}^{2 \omega}= & d_{15} \cos \phi \sin 2 \theta\left(E^{\omega}\right)^{2}, \\
P_{y}^{2 \omega}= & d_{24} \sin \phi \sin 2 \theta\left(E^{\omega}\right)^{2}, \\
P_{z}^{2 \omega}= & {\left[d_{33} \cos ^{2} \theta+\left(d_{15} \cos ^{2} \phi+d_{24} \sin ^{2} \phi\right) \sin ^{2} \theta\right] } \\
& \times\left(E^{\omega}\right)^{2} .
\end{aligned}
$$

For comparison with the angular dependence of the measured SH signals Eqs. (5) have to be squared because the intensities are registered. Furthermore, it has to be noted that the actual measured SHG intensity has an incidence angle dependence $[\propto \cos (\theta)]$ due to the increasing ellipticity of the incident fundamental wave with an increase in $\theta$.

\section{RESULTS AND DISCUSSION}

Figure 3 shows the result of a typical SHG experiment on the BBiT sample described above. Here $p$-polarized light with the laser fundamental wavelength $(800 \mathrm{~nm})$ was irradiated into a region with a considerable density of non-coriented crystallites. In Fig. 3 the monitored SH intensity is plotted on a gray scale (where dark regions refer to low $\mathrm{SH}$ intensity, and light ones to high SH intensity) versus the azimuth angle $\phi$ and the incidence angle $\theta$. The upper plot corresponds to $p$-polarized observation (abbreviated $p-p$ ) and the lower one to the $s$ component $(p-s)$ of the $\mathrm{SH}$ intensity observed. For both polarization geometries rather strong SHG signals are observed, the maximum being a factor of 3 larger for the $p-p$ situation as opposed to that in the $p-s$ case. A common feature of $p-p$ and $p-s$ geometry is the rather simple variation of the intensity (integrated over $\phi$ ) with the incidence angle $\theta$ : starting with low SH intensity 
at $\theta=20^{\circ}$ the signal increases with an increase in $\theta$ to a maximum (around $60^{\circ}$ for $p-p$ geometry and $40^{\circ}$ for $p-s$ geometry) and then decreases again. This decrease at large incidence angles is apparently a result of the general $\cos (\theta)$ dependence of the SHG intensity.

The variation with sample rotation (azimuthal angle $\phi$ ) is more complicated: in both polarization geometries fourfold symmetry, i.e., $90^{\circ}$ periodicity, is clearly visible. A closer inspection reveals that in both cases two series of maxima can be distinguished: for the $p-s$ geometry (lower graph) the azimuthal dependence of the SH intensity observed can be decomposed into two series of maxima located typically at the same incidence angle of $\theta \approx 40^{\circ}$, where the stronger peaks are found at $\phi \approx 25^{\circ}+n \cdot 90^{\circ}(n=0, \ldots, 3)$ and the weaker ones at $\phi \approx 80^{\circ}+n \cdot 90^{\circ}$. In the case of the $p-p$ geometry (upper graph) the more prominent series of maxima is located at $\phi \approx 5^{\circ}+n \cdot 90^{\circ}\left(\theta \approx 55^{\circ}\right)$, while an additional series of less pronounced maxima can be seen at $\phi$ $\approx 50^{\circ}+n \cdot 90^{\circ}\left(\theta \approx 65^{\circ}\right)$. For the latter series, the peaks at $50^{\circ}$ and $320^{\circ}$ appear to be weaker than the other two, so here an additional contribution with lower symmetry (twofold or even onefold) seems to be present.

In order to find a reasonable assignment for the observed angular modulation of the $\mathrm{SH}$ signal, control experiments were performed in regions that showed only the $c$-axis oriented BBiT background. In these regions also considerable SH intensity was observed, with the $\theta$ dependence being very similar to that shown in Fig. 3 , but no $\phi$ dependence at all within experimental accuracy. This observation allows us to conclude that any variations of the SHG connected with the azimuthal rotation of our samples can be attributed to the non- $c$-axis oriented grains. More details can be extracted with regard to Eqs. (5) and the orientational distribution of BBiT crystallites as was shown in the electron microscope pictures presented in Fig. 1. For a (perfect) single crystal with $m m 2$ symmetry Eqs. (5) yield a $I(\phi) \propto \sin ^{2}(2 \phi)$ dependence, i.e., $90^{\circ}$ periodicity of the observed SH intensity for the $p-s$ geometry, while the signal observed in the $p-p$ geometry is characterized by $I \propto\left(d_{15} \cos ^{2} \phi+d_{24} \sin ^{2} \phi\right)^{2}$, which means $180^{\circ}$ periodicity (or a constant signal in the case of $d_{15}=d_{24}$ ). Since both types of crystallites are found in two azimuthal orientations perpendicular to each other, the observed fourfold symmetry can be explained in principle on the basis of Eqs. (5). Additionally, taking into account the fact that the edges of the (110)-oriented crystallites form angles of $45^{\circ}$ with the edges of the (100)-oriented ones, even the occurrence of the two "series" of maxima seen in Fig. 3 can be understood qualitatively. The presence of a contribution by the lower symmetry found in the data in $p-p$ geometry may be taken as an indication that there is not an equal number of (110) crystallites in the different possible azimuthal orientations.

Overall it seems quite reasonable to attribute the two periodic series of maxima observed in the SHG azimuthal dependence to the two different types of BBiT grains. Experimental evidence for this assignment is found by measuring the SH signals at sample locations with different contents of individual crystallite types; because the average grain distribution only varies on a length scale of millimeters across
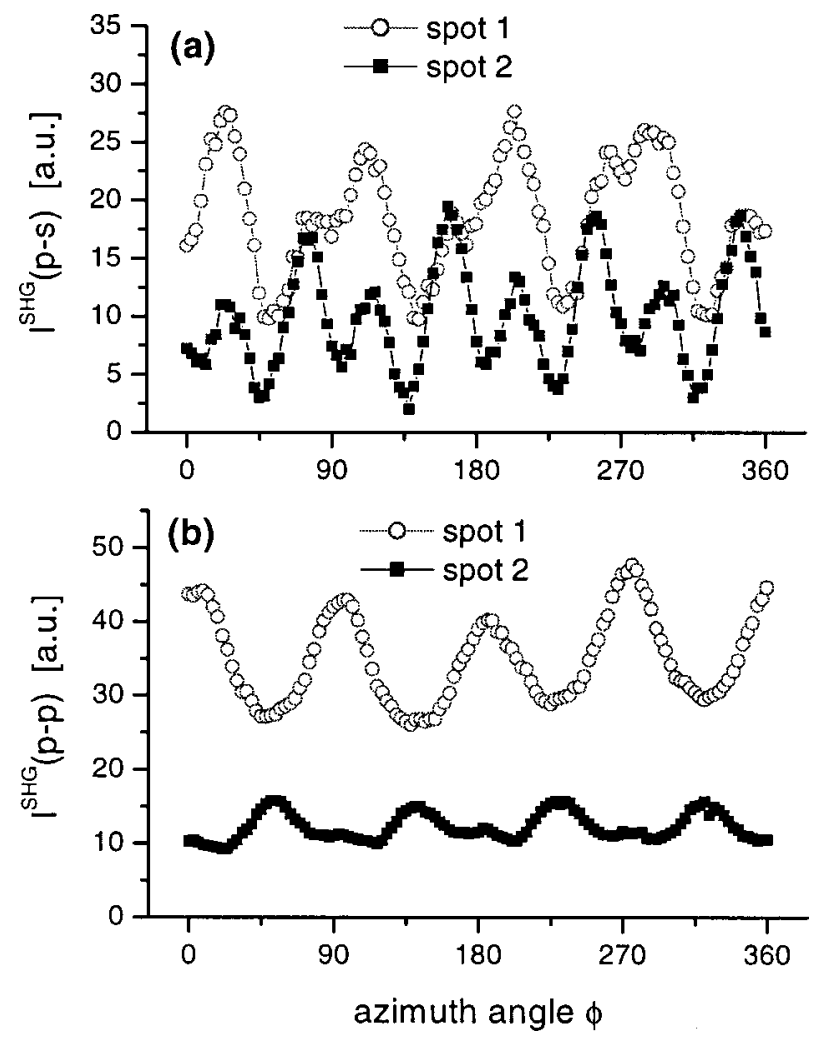

FIG. 4. Azimuthal dependence of the SH intensity $(\lambda=400 \mathrm{~nm})$ measured at incidence angle $\theta=30^{\circ}$ at two different spots on the sample corresponding to the positions in Figs. 1(a) [spot (1), open circles] and 1(b) [spot (2), closed squares). (a) $p-s$ geometry and (b) $p-p$ geometry.

the Si substrate wafer, it is not difficult to adjust the laser spot to a sample area with known distribution (already measured by SEM). An example of these results is presented in Fig. 4, where only the azimuthal dependencies of $I(p-s)$ [Fig. 4(a)] and $I(p-p)$ [Fig. 4(b)] for an incidence angle of $\theta=30^{\circ}$ are plotted for two different sample positions corresponding to the ones shown in the SEM pictures of Fig. 1. The open circles represent a spot (1) where the rectangularshaped grains are dominant [cf. to Fig. 1(a)], while at the position of spot (2) [corresponding to the situation shown in Fig. 1(b)] the square-shaped ones are in the majority. Quite obviously in both plots the amplitude ratio of the already discussed two $90^{\circ}$-spaced series of intensity maxima is reversed going from spot (1) to spot (2). This observation provides strong experimental evidence that we can assign the more prominent maxima observed at spot (1) to $\mathrm{SH}$ photons generated in the (110)-oriented (rectangular) crystallites, and the dominant peaks observed at spot (2) to the (100)-oriented (square-shaped) crystallites. Due to this unambiguous empirical correlation the technique presented appears to be well suited for purely optical detection of the nanoscopic texture of epitaxially produced ferroelectric thin films (or, more generally, for any other noncentrosymmetric crystalline material), and could thus in principle be used for in situ control of the growth process.

Beyond this finding it would be very interesting to analyze the signals measured in more detail in order to extract the nonzero tensor elements of the second order optical nonlinearity, and in particular the influence of ferroelectric po- 
larization on these parameters. However, a simple mathematical consideration reveals that Eqs. (5) (and thus the description used to derive them) is not sufficient to quantitatively model the $\phi$ dependence of the SHG measurements presented: the highest power of $\sin \phi$ (or $\cos \phi$ ) that in Eqs. (5) is the fourth power, which can not reproduce more than fourfold symmetry, even if contributions from the differently oriented crystallites are superimposed. Since it is not the aim of this work to present a comprehensive quantitative evaluation of the measured SHG, we will only discuss the important points, which a correct description would have to account for. (i) The crystallites, being mainly responsible for the SHG, of the order of $100 \mathrm{~nm}$ in size and embedded in a $c$-axis oriented layer; therefore a considerable part of the volume of such a grain will show lattice distortions, i.e., the prerequisite of perfect $m m 2$ symmetry underlying Eqs. (5) is most probably not valid. (ii) It was demonstrated recently that interference effects can play an important role in studying thin films with the help of optical second-harmonic generation. ${ }^{30}$ (iii) There is a need to discuss the question of the actual direction of the propagation of light in a crystal that has dimensions that are clearly less than both the fundamental and second-harmonic wavelengths. (iv) At room temperature, $\mathrm{BBiT}$ is in the ferroelectric phase, and this means that the present remnant polarization may in principle create additional, field-induced tensor elements in the optical nonlinearity. It was shown very recently for $\mathrm{Pb}(\mathrm{ZrTi}) \mathrm{O}_{3}$ films that these elements can be used to detect ferroelectric polarization by SHG experiments; ${ }^{31}$ however, since the electric field-induced terms are usually much smaller than crystallographic ones, ${ }^{31}$ this is not expected to be important for interpretation of the data presented. It will, however, be an interesting aspect of future experiments where one may attempt to also investigate the ferroelectric characteristics of these BBiT films and of other Aurivillius phases with the help of SHG.

\section{SUMMARY}

Second-harmonic generation on epitaxial ferroelectric thin films of $\mathrm{BaBi}_{4} \mathrm{Ti}_{4} \mathrm{O}_{15}$ was investigated by full angular and polarization resolution. The results showed a clear correlation between the azimuthal dependence of the SH intensity measured and the nanoscopic texture of the samples, which was studied with the help of electron microscopy. In particular, the relative densities of (110)- and (100)-oriented grains at the surface correspond to the amplitudes of different $90^{\circ}$ periodic series of $\mathrm{SH}$ maxima, and they can be separated from the "isotropic" (i.e., constant versus azimuthal sample rotation) background because of the $c$-axis oriented main layer. This unambiguous correspondence makes this
SH method appear to be well suited as a nondestructive optical in situ technique by which to control the film orientation and thickness during growth.

\section{ACKNOWLEDGMENT}

The authors gratefully acknowledge financial support by the Deutsche Forschungsgemeinschaft through the Forschergruppe 404 "Oxidic Interfaces."

${ }^{1}$ J. F. Scott, Ferroelectr. Rev. 1, 1 (1998).

${ }^{2}$ O. Auciello, J. F. Scott, and R. Ramesh, Phys. Today 51, 22 (1998).

${ }^{3}$ D. Damjanovic, Rep. Prog. Phys. 61, 1267 (1998).

${ }^{4}$ R. Ramesh, A. Inam, W. K. Chan, B. Wilkens, K. Myers, K. Remschnig, D. L. Hart, and J. M. Tarascon, Science 252, 944 (1991).

${ }^{5}$ C. A. Paz de Araujo, J. D. Cuchiaro, L. D. McMillan, M. C. Scott, and J. F. Scott, Nature (London) 374, 627 (1995).

${ }^{6}$ J. F. Scott and C. A. Paz de Araujo, Science 246, 1400 (1989).

${ }^{7}$ Symetrix Corp., Int. Patent No. H01L27/115, 21/320529/92 (1992).

${ }^{8}$ B. Aurivillius, Ark. Kemi 2, 519 (1950) (in English); E. C. Subba Rao, J. Am. Ceram. Soc. 45, 166 (1962).

${ }^{9}$ B. Aurivillius and P. H. Fang, Phys. Rev. 126, 893 (1962); P. H. Fang, C. R. Robbins, and B. Aurivillius, ibid. 126, 892 (1962).

${ }^{10}$ E. C. Subbarao, J. Phys. Chem. Solids 23, 665 (1962).

${ }^{11}$ S. E. Cummins and L. E. Cross, Appl. Phys. Lett. 10, 14 (1967).

${ }^{12}$ A. Pignolet, K. M. Satyalakshmi, M. Alexe, N. D. Zakharov, C. Harnagea, S. Senz, D. Hesse, and U. Gösele, Integr. Ferroelectr. 26, 723 (1999).

${ }^{13}$ A. Pignolet, C. Schäfer, K. M. Satyalakshmi, C. Harnagea, D. Hesse, and U. Gösele, Appl. Phys. A: Mater. Sci. Process. A70, 283 (2000).

${ }^{14}$ E. C. Subbarao, Phys. Rev. 122, 804 (1961).

${ }^{15}$ H. Tabata, M. Hamada, and T. Kawai, Mater. Res. Soc. Symp. Proc. 401, 73 (1996).

${ }^{16}$ H. Tabata, T. Yanagita, and T. Kawai, IEICE Trans. Electron. E81-C, 566 (1998).

${ }^{17}$ E. D. Mishina, N. E. Sherstyuk, T. V. Misyurae, A. S. Sigov, A. M. Grishin, Th. Rasing, and O. A. Aksipetrov, Thin Solid Films 336, 291 (1998).

${ }^{18}$ B. Bihari, J. Kumar, G. T. Stauf, P. C. Van Buskirk, and C. S. Hwang, J. Appl. Phys. 76, 1169 (1994).

${ }^{19}$ L. D. Rotter, D. L. Kaiser, and M. D. Vaudin, Appl. Phys. Lett. 68, 310 (1996).

${ }^{20}$ T. Zhao, Z.-H. Chen, F. Chen, W.-S. Shi, H.-B. Lu, and G.-Z. Yang, Phys. Rev. B 60, 1697 (1999).

${ }^{21}$ Y. Barad, J. Lettieri, C. D. Theis, D. G. Schlom, V. Gopalan, J. C. Jiang, and X. Q. Pan, J. Appl. Phys. 89, 1387 (2001).

${ }^{22}$ K. M. Satyalakshmi, M. Alexe, A. Pignolet, N. D. Zakharov, C. Harnagea, S. Senz, and D. Hesse, Appl. Phys. Lett. 74, 603 (1999).

${ }^{23}$ K. M. Satyalakshmi, A. Pignolet, M. Alexe, N. D. Zakharov, C. Harnagea, S. Senz, S. Reichelt, D. Hesse, and U. Gösele, Mater. Res. Soc. Symp. Proc. 541, 205 (1999).

${ }^{24}$ S. K. Kim, M. Myayama, and H. Yanagida, J. Ceram. Soc. Jpn. 102, 722 (1994).

${ }^{25}$ W. N. Herman and L. M. Hayden, J. Opt. Soc. Am. B 12, 416 (1995).

${ }^{26}$ V. G. Dmitriev, G. G. Gurzadyan, and D. N. Nikogosyan, Handbook of Nonlinear Optical Crystals (Springer, Berlin, 1991).

${ }^{27}$ S. V. Popov, Susceptibility Tensors for Nonlinear Optics (IOP, Bristol, 1995).

${ }^{28}$ A. Yariv, Quantum Electronics (Wiley, New York, 1990).

${ }^{29}$ D. A. Kleinman, Phys. Rev. 126, 1977 (1962).

${ }^{30}$ F. Balzer and H.-G. Rubahn, Opt. Commun. 185, 493 (2000).

${ }^{31}$ E. D. Mishina, N. E. Sherstyuk, E. P. Pevtsov, K. A. Vorotilov, A. S. Sigov, M. P. Moret, S. A. Rossinger, P. K. Larsen, and T. Rasing, Appl. Phys. Lett. 78, 796 (2001). 